\title{
Cold-electron bolometer as a photon-noise-limited detector with on-chip electron self-cooling
}

\author{
$\underline{\text { L.S. Kuzmin }}^{1,2}$, A.L. Pankratov ${ }^{1,3}$, A.V. Gordeeva ${ }^{1,3}$, V.O. Zbrozhek ${ }^{1}$, \\ A.V. Blagodatkin ${ }^{1,3}$, L.S. Revin ${ }^{1,3}$ \\ ${ }^{1}$ Nizhny Novgorod State Technical University n.a. R.E. Alekseev, Nizhny Novgorod, Russia \\ ${ }^{2}$ Chalmers University of Technology, Gothenburg, Sweden, leonid.kuzmin@chalmers.se \\ ${ }^{3}$ Institute for Physics of Microstructures of RAS, Nizhny Novgorod, Russia
}

After discovering the electron cooling by Superconductor-Insulator-Normal metal (SIN) tunnel junctions, significant efforts were applied to obtain an effective on-chip cooler for $\mathrm{mm} / \mathrm{IR}$ detectors. We have developed a Cold-Electron Bolometer (CEB) with effective direct electron self-cooling of the absorber [1], [2]. A photon-noise-limited pixel, consisting of an array of CEBs with self-cooling, is realized for OLIMPO Balloon Telescope for incoming power $P_{\text {in }}$ up to $60 \mathrm{pW}$ at phonon temperature $T_{p h}=310 \mathrm{mK}$. Operation of the bolometer at electron temperature less than phonon temperature significantly increases its sensitivity so that the noise equivalent power goes beyond the photon-noise-limited mode, which means that the internal bolometer noise is smaller than the noise of incoming signal [3]. We demonstrate the electron cooling from 310 to $120 \mathrm{mK}$ without signal and from 410 to $225 \mathrm{mK}$ for Pin $=60 \mathrm{pW}$ at $350 \mathrm{GHz}$. The proposed technology is a potential replacement for the high-cost dilution refrigerators for space applications.

The cold electron bolometer is a SINIS structure, integrated in a planar antenna, designed to absorb terahertz radiation at certain frequency. Its SEM image is shown in Fig.1. The normal metal is made of aluminum with suppressed superconductivity, the tunnel barrier is formed by oxidation of aluminum and the superconducting electrodes are made of clean aluminum. All layers are deposited on a Si substrate by method of shadow evaporation. The volume of the normal absorber is just $0.02 \mu \mathrm{m}^{3}$.

A single CEB can absorb up to $0.3-0.5 \mathrm{pW}$ of power before it saturates. In order to fulfill the requirements of high power load for OLIMPO mission, we connected 192 single CEBs in one array. Each bolometer is integrated in a dipole antenna. The array is tuned to have its maximal absorption at $350 \mathrm{GHz}$. The experiments have shown that the efficiency of absorption for fabricated samples is more than $50 \%$.

The cold electron bolometer is well described by the heat balance equation for normal absorber with resistance $R_{N}$ :

$$
P_{a b s}+I^{2} R_{N}+V^{2} / R_{\text {leak }}=2 * P_{C O O L}+\sum \mathrm{V}\left(T_{e}^{2}-T_{p h}{ }^{2}\right),(1)
$$

where $I$ - tunnel current, $V$ - voltage drop across one SIN junction, $P_{a b s}$ - absorbed power, $R_{\text {leak }}$ - leakage resistance of SIN junctions, $P_{C O O L}$ - cooling power of SIN junctions, $\sum$ - electron-phonon constant, $V$ - volume of the absorber, $T_{e}$ and $T_{p h}$ are electron and phonon temperatures of the absorber, respectively.

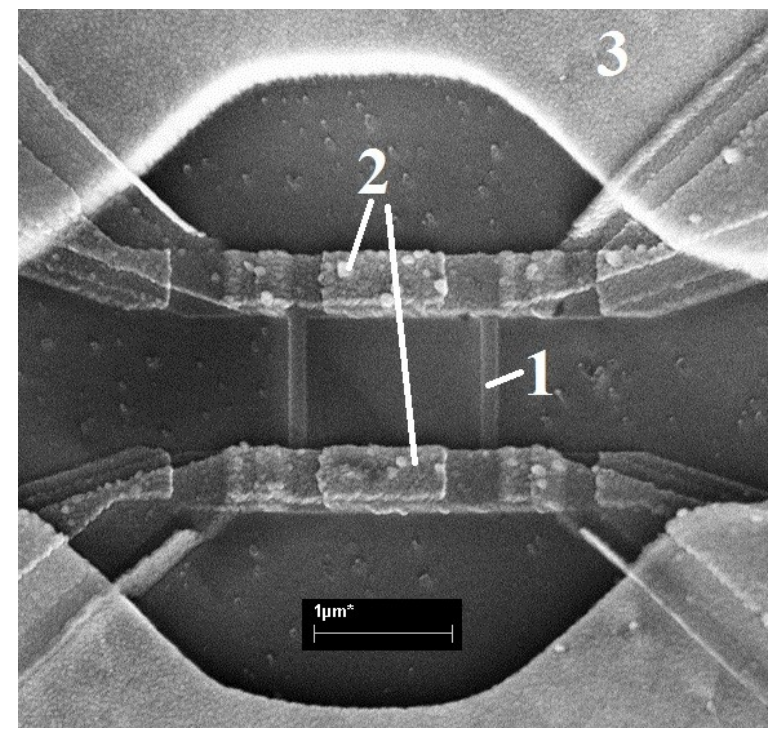

Fig. 1. SEM image of a single cold electron bolometer integrated in a gold dipole antenna. 1 - normal absorber, 2 SIN junction, 3 - antenna.

The fabricated samples have been cooled in a dilution cryostat down to $310 \mathrm{mK}$ and irradiated by a black body source through a set of quasi-optical filters for $350 \mathrm{GHz}$. The voltage response and voltage noise at several black body temperatures from $2 \mathrm{~K}$ to $46 \mathrm{~K}$ have been measured, using room temperature ultralow noise amplifiers AD745.

We have compared the experimental IV-curves with Eq. (1) and found that our samples can be fitted very well with the following parameters: critical temperature of superconductor $1.24 \mathrm{~K}$, phonon temperature $310 \mathrm{mK}, \sum=1.3 \mathrm{nW} / \mathrm{K}^{5} / \mu \mathrm{m}^{3}$, normal resistance of SIN junction $1.6 \mathrm{k} \Omega, R_{\text {leak }}=25 \mathrm{M} \Omega, R_{N}=50 \Omega$.

The main characteristic of any detector is the noise equivalent power (NEP). The modern bolometers for astronomical applications are required to have intrinsic NEP less than the photon NEP of incoming signal, i.e. the detector has to be limited by the photon noise. We have shown in a set of our experiments, that we indeed can see the photon noise in our detectors in a broad range of incoming powers from 20 to $60 \mathrm{pW}$.

The NEP of the bolometer array is shown in Fig.2 for the case of zero incoming power. One can see good agreement between measured and theoretical 
values. The optimal operational point of the bolometer can be chosen at the minimal NEP around $12 \mathrm{mV}$. At this voltage the largest contribution to the bolometer NEP comes from the noise of SIN contacts, which is the sum of shot noise of current through a tunnel junction, noise of heat flow and correlation between them. Next largest NEP component is amplifier NEP. Whereas NEP due to electron-phonon interaction is significantly smaller than other NEP components and can be even disregarded.

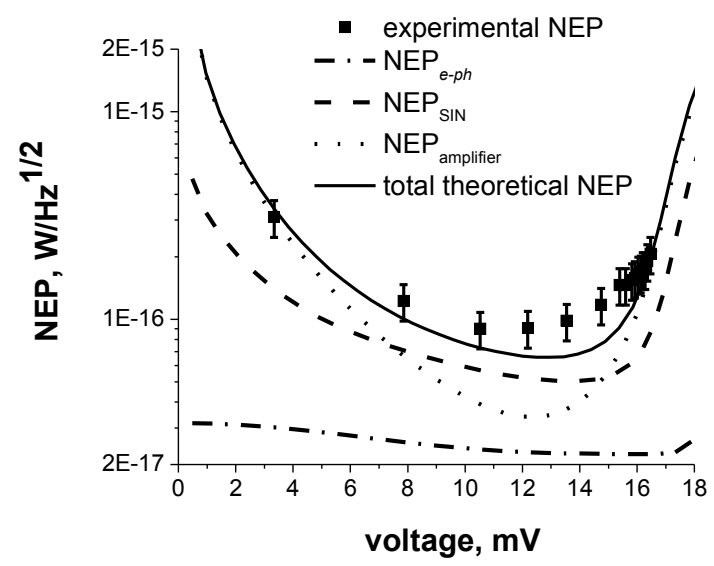

Fig. 2. Noise equivalent power versus voltage on the bolometer array with zero power load. The phonon temperature $310 \mathrm{mK}$.

In Fig. 3 we show NEP of the same bolometer array but for high power load $60 \mathrm{pW}$, which corresponds to $32 \mathrm{pW}$ of absorbed power. Now we have one more NEP component - photon noise of absorbed power (straight gray line in Fig. 3). One can see that at voltages 11-12 $\mathrm{mV}$ the photon NEP goes a bit higher that all other NEP components together, which means that the detector is photon limited.

Analyzing our data, we found that for black body temperatures above $20 \mathrm{~K}$, the phonon temperature of the sample also increases and can be higher than the cryostat temperature. For example for Fig. 3 the phonon temperature was $380 \mathrm{mK}$. But even with this overheating the samples show photon limited mode of operation.

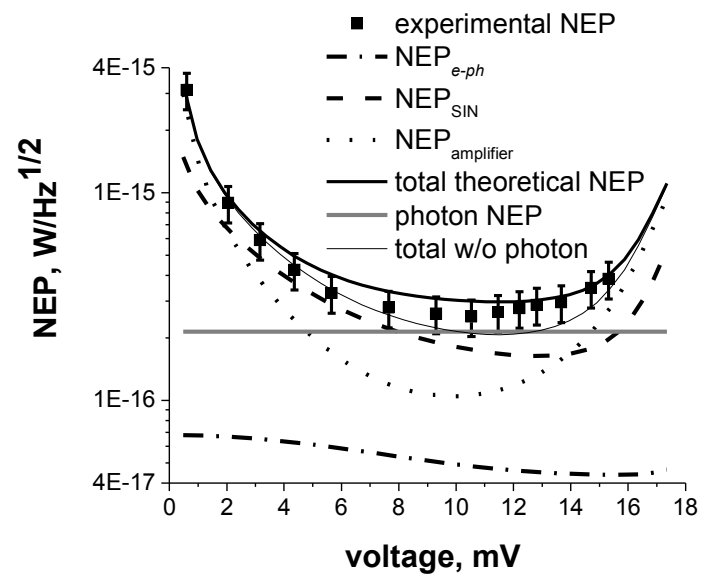

Fig. 3. Noise equivalent power versus voltage on the bolometer array. Radiated power $60 \mathrm{pW}$. Absorbed power 32 $\mathrm{pW}$.

Authors would like to thank M. Tarasov and A. Gunbina for help in the sample fabrication, E. Skorokhodov for making SEM images of the samples. The samples were fabricated in the Chalmers anotechnology Center. The facilities of the Center of Cryogenic Nanoelectronics of NNSTU and Common Research Center "Physics and technology of micro- and nanostructures" of IPM RAS were used.

The work is supported by Russian Science Foundation (Project 16-19-10468).

\section{References}

1. Kuzmin, L. An Array of Cold-Electron Bolometers with SIN Tunnel Junctions and JFET readout for Cosmology Instruments // Journal of Physics: Conference Series, 8th European Conference on Applied Superconductivity. 2008. V. 97.

2. Kuzmin, L; Golubev, D. On the concept of an optimal hot-electron bolometer with NIS tunnel junctions // Physica C-Superconductivity and Its Applications. 2002. V. 372. P. 378-382.

3. Gordeeva,A.V., et al. Observation of photon noise by cold-electron bolometers // Appl. Phys. Lett. 2017. V. 110. P. 162603. 